\title{
因特网 AS 级拓扑的健壮性测度与健壮性演化
}

\author{
卢锡城 ${ }^{(1)}$ 邓文平 (2)*, 朱培栋 ${ }^{(1)}$, 胡晓峰 ${ }^{\text {(1) }}$ \\ (1) 国防科技大学计算机学院, 长沙 410073 \\ (2) 国防科技大学信息中心, 长沙 410073 \\ * 通信作者. E-mail: wpdeng@nudt.edu.cn
}

收稿日期: 2010-11-22; 接受日期: 2011-12-09

国家自然科学基金 (批准号: 61070199, 61170285, 61170286) 和国家高技术研究发展计划 (批准号: 2008AA01Z407) 资助项目

摘要以自治系统 (autonomous system, AS) 为基本组成单元的域间路由系统是因特网 (Internet) 的 核心组成部分, 研究 Internet AS 级拓扑的健壮性对于整个 Internet 的健壮性设计具有重要意义. 2003 年的北美停电事故与 2006 年、2010 年的台湾地震表明, 当今 Internet 的健壮性仍亟待增强. 本文结 合基本的拓扑理论与实际的路由策略约束, 利用 Internet AS 级拓扑的层次特性, 从 “单个 AS” 和 “全 局拓扑”两个不同的层面研究 AS 级拓扑的健壮性测度与健壮性演化: 1) 提出了对单个 AS 的健壮性 测度的方法; 2) 对全局 AS 级拓扑的健壮性测度提出了 $k$ 容错模型——任意 $k$ 个 AS 级的节点/链 路故障不影响任何其他两个 $\mathrm{AS}$ 之间的连通性; 3) 基于健壮性测度方法和 $k$ 容错模型, 提出了健壮 性演化的机制与方法. 对当今 Internet AS 级拓扑的统计分析表明: 1) $25.8 \%$ 的 AS 节点还不能承受 1 个 AS 级的链路故障, $26.4 \%$ 的 AS 节点还不能承受 1 个 AS 节点故障; 2) 将现有的 AS 级拓扑演化为 $k$ 容错拓扑不仅能保证任意节点对之间可达性的 $k$ 容错, 在故障数超过 $k$ 的情况下, $k$ 容错拓扑的健 壮性也显著优于原拓扑.

关键词域间路由系统 自治系统 健壮性 $k$ 容错 拓扑

\section{1 引言}

域间路由系统是 Internet 路由系统的核心, 由数万个分布在全球的自治系统 (autonomous system, AS) 和它们之间的连接构成. 每个自治系统属于一个特定的组织机构或网络运营商 (internet service provider, ISP), 包含了处于同一机构相同策略管理之下的若干网络和路由器, 由一个唯一确定的非负 整数所标识. 域间路由系统的核心则是边界网关协议 (border gateway protocol, BGP) ${ }^{[1]}$, 它工作在各 个 AS 的边界路由器之间并保证各自治系统之间的连通性. 一条 BGP 路由给出了通往一个目的网络 (prefix) 所要穿越的 AS 路径. 根据 AS 路径所包含的 AS 节点和 AS 之间的连接, 可构建出 Internet 在 AS 级的拓扑结构.

从多方面看, Internet 域间路由系统从成长之初到现在一直都面临随机故障、网络攻击和自然灾 害的威胁, 域间路由系统健壮性仍然是下一代 Internet 面临的关键问题. 文献 [2] 指出, 超过 $50 \%$ 的 VoIP 通话故障是由 BGP 故障所引发的. 2003 年 8 月北美联合电网发生的大停电事故 ${ }^{[3]}, 2006$ 年 
12 月和 2010 年 3 月的台湾地震等都对全球网络的连通性产生了严重影响 [4]. 由此可见, 当前路由系 统在容错性与健壮性方面仍然存在明显的不足. 而这一问题的根源之一在于 Internet 的拓扑缺乏宏 观全局规划, 节点和链路缺乏足够的圥余备份. 我们针对现有 AS 级拓扑数据所作的统计表明, 超过 $20 \%$ 的 AS 仅与一个上游的 $\mathrm{AS}$ 连接, 这些 AS 的网络连通性完全依赖于单个上游服务提供商. 简单 地在 AS 之间增加备份链路或者以多宿主 (multi-homing) 方式增加上游服务提供商数目固然能增强 域间路由系统个别 AS 的网络健壮性, 但是, 新增一条上游备份链路意味着 ISP 投入的增加并且关系 到多方的商业利益. 由此引发了两个问题: 1) 当今 Internet 域间路由系统到底有多健壮;2) 怎样有效 引导 Internet 的健壮性演化.

早期的研究纯粹从图论或者复杂网络的角度来研究域间路由系统 AS 级拓扑的特征参数与健壮 性. 这些研究工作一般使用简单图 (无向图或有向图) 来表示复杂网络拓扑, 研究 Internet 所具备的 宏观统计特性, 比如, Siganos 等 ${ }^{[5]}$ 在 1999 年发现 Internet 拓扑结构存在幕律分布特性, 从而引导 Internet 拓扑研究工作进入无标度复杂网络研究时代. 文献 [6] 基于 Internet 拓扑结构的无尺度特性进 一步阐述了 Internet 的 “健壮亦脆弱 (robust yet fragile)” 的双重特性, 即对随机故障的健壮性和对定 点攻击的脆弱性. 虽然这些研究结果对于 Internet AS 级拓扑健壮性的分析具有重要的参考作用, 但 它们没有考虑 Internet AS 级拓扑结构自身的独特性与路由策略的约束. 域间路由是一种基于策略的 路由, AS 之间的连接 (路由输出策略与流量转发) 基于 ISP 之间的商业关系协商, 由 AS 双边的边界 路由器建立 BGP 会话来实现. 因而, 域间路由系统的拓扑结构既包括物理意义上的 BGP 路由器互连 结构, 也包括逻辑意义上的 AS 级互连结构, 前者刻画了 Internet AS 之间的物理连通性, 后者刻画了 Internet AS 之间真正的逻辑可达性.

本文从 AS 商业关系模型出发, 利用 Internet AS 级拓扑的层次特性 ${ }^{[7,8]}$, 从 “单个 AS” 和 “全局 拓扑” 两个不同的层面研究 Internet AS 级拓扑的健壮性测度与健壮性演化. 提出了对单个 AS 健壮 性的测度指标—单个 AS 所拥有的到达顶级 AS 的节点/边不相交的上坡路径数目, 利用该指标来 评测给定 AS 对于 AS 级的节点/链路故障的健壮性. 对于全局 AS 级拓扑, 本文从域间路由系统 AS 级拓扑的 $k$ 容错判定问题出发, 利用 AS 级拓扑固有的层次结构, 提出了 $k$ 容错指标, 即任意 $k$ 个 AS 级的节点/链路故障不影响任何其他两个 AS 之间的连通性. 基于以上健壮性测度指标, 提出了健壮性 演化的机制与方法. 最后, 采用 $\mathrm{CAIDA}^{1}$ 和 $\mathrm{AquaLab}^{2}$ 提供的真实拓扑数据, 对本文的方法进行了评 估. 对当今 Internet AS 级拓扑的统计分析表明: 1) 有 $25.8 \%$ 的 AS 节点还不能承受 1 个 AS 级的链 路故障, $26.4 \%$ 的 AS 节点还不能承受 1 个 AS 节点故障; 2) 将现有的 AS 级拓扑演化为 $k$ 容错拓扑 不仅能保证任意节点对可达性的 $k$ 容错, 在故障数超过 $k$ 的情况下, $k$ 容错拓扑的健壮性也显著优于 原拓扑.

本文的第 2 节给出了相关的研究背景; 第 3 节主要描述了对 “单个 AS” 的健壮性测度与健壮性 演化; 第 4 节则对 “全局拓扑” 的健壮性测度与健壮性演化做了论述, 并重点描述了全局 AS 级拓扑的 $k$ 容错判定; 第 5 节给出了相关的实验分析; 最后, 第 6 节对全文进行了总结.

\section{2 研究背景}

Internet 以 AS 为界, 采用层次式路由结构. AS 内部使用域内路由协议, 如 RIP, IS-IS 和 OSPF; AS 之间使用域间路由协议 BGP. 域内路由协议与域间路由协议通过路由重发布技术交换路由信息.

1) Http://as-rank.caida.org/data

2) Http://aqualab.cs.northwestern.edu/projects/SidewalkEnds.html 
自治系统之间在商业关系的约束之下实现流量的转发与路由的相互通告. 同时, 自治系统以及它们之 间的商业关系所构成的 Internet AS 级拓扑结构在宏观上呈现出了一些独特的性质, 比如 AS 路径的 “无谷底” 特性与层次结构特性.

\section{$2.1 \mathrm{AS}$ 商业关系与 $\mathrm{AS}$ 级拓扑}

$\mathrm{AS}$ 之间存在 4 种基本的商业关系 ${ }^{[9]}$ : 提供商 - 客户关系 (provider-customer, p2c)、客户 - 提供 商关系 (customer-provider, c2p)、对等关系 (peer-peer, p2p) 以及同胞关系 (sibling-sibling, s2s). AS 之 间的商业关系的存在深刻地影响着 Internet 端到端的路由. 在一个 $\mathrm{p} 2 \mathrm{c}$ 的关系中, 客户 AS 需要付费 给提供商 $\mathrm{AS}$, 提供商帮客户转发流量, 而通常客户 AS 的规模都较提供商 $\mathrm{AS}$ 明显要小得多, 这是一 种非对称的关系; 在一个 $\mathrm{p} 2 \mathrm{p}$ 的关系中, 双方是对称互惠的, 仅用于互惠免费转发来自各自内部或各 自客户流量, 不能通过对方访问外部的网络; 而在一个 $\mathrm{s} 2 \mathrm{~s}$ 的关系中, 双方可能属于同一个上游机构, 地位相当, 完全互惠, 通常可互相作为备份. 基于 AS 之间的商业关系, Internet 可被抽象为一个关系 类型图 (type-of-relationship graph) $G=(V, E, R)^{[10]}$, 其中, $V$ 是 $\mathrm{AS}$ 集合, $E$ 是 $\mathrm{AS}$ 之间的连接集合, $R$ 是 $E$ 到集合 $\{\mathrm{p} 2 \mathrm{c}, \mathrm{c} 2 \mathrm{p}, \mathrm{p} 2 \mathrm{p}, \mathrm{s} 2 \mathrm{~s}\}$ 的映射，即每条边对应一种具体的商业关系类型.

受商业关系的约束, 在与一个提供商或者对等体交换路由信息时, 一个自治系统可以输出自己的、 其客户的以及同胞的路由, 但是不能输出从其他提供商或对等 AS 学到的 BGP 路由; 而在与一个客 户或者同胞交换路由信息时, 一个自治系统可以输出自己学到的所有路由, 包括从其客户、同胞、提 供商以及对等 AS 学到的 BGP 路由. 基于 BGP 的路由输出策略, 一条有效的 AS 路径应满足 “无谷 底” 特性 ${ }^{[9]}$ :

定义 1 (“无谷底” 特性, valley-free property) 一个 AS 不能在它的两个提供商 AS (对等 AS) 之 间转发流量, 即在穿越了一条 $\mathrm{p} 2 \mathrm{c}$ 或者 $\mathrm{p} 2 \mathrm{p}$ 连接之后, 一条 AS 路径不能再穿越一条 $\mathrm{c} 2 \mathrm{p}$ 或者 $\mathrm{p} 2 \mathrm{p}$ 连接.

特别地, 由于 $\mathrm{s} 2 \mathrm{~s}$ 类型的商业关系所占的比例非常小 (根据 CAIDA 提供的商业关系数据, s2s 所 占的比例还不到 $0.3 \%$ ), 并且在很多情况下可等同于 $\mathrm{p} 2 \mathrm{p}$ (都具有对称性), 之前的研究 ${ }^{[11,12]}$ 都把关系 类型图中的 $\mathrm{s} 2 \mathrm{~s}$ 连接忽略不计或者视为 $\mathrm{p} 2 \mathrm{p}$ 连接. 本文也作了相似的简化: 在考虑 $\mathrm{AS}$ 连通性时, 把 $s 2 \mathrm{~s}$ 连接当成 $\mathrm{p} 2 \mathrm{p}$ 连接对待. 更为直观地, 我们把一条 $\mathrm{c} 2 \mathrm{p}$ 连接看作是一条从客户到提供商的有向边 并记为 $\rightarrow$, 把 $\mathrm{p} 2 \mathrm{p}$ 记为 $\leftrightarrow$. 基于 “无谷底” 这一基本约束, $\mathrm{Gao}^{[9]}$ 提出了两个基本的概念用以描述有 效的路径模式: 上坡路径和下坡路径. 一条上坡路径 (下坡) 由一个 $\mathrm{c} 2 \mathrm{p}(\mathrm{p} 2 \mathrm{c})$ 的序列构成, 即一个源 AS 节点经过一系列的 $\mathrm{c} 2 \mathrm{p}$ (p2c) 连接到达一个目的 AS 节点的过程称为上坡 (下坡). 基于这两个基本 概念, 一条有效的 AS 路径无外乎以下 6 种模式 ${ }^{[9]}$ : 1) 上坡路径; 2) 下坡路径; 3) 上坡路径 +一条对 等连接 (p2p)；4) 一条 p2p 连接 + 下坡路径; 5) 上坡路径 + 下坡路径; 6) 上坡路径 + 一条 p2p 连接 + 下坡路径. 以上 6 种模式之外的任何 AS 路径都必然违背 “无谷底” 特性, 因而是无效的.

\section{$2.2 \mathrm{AS}$ 级拓扑的层次特性}

基于 AS 商业关系, 文献 [13] 进一步论述了 AS 级拓扑在商业关系约束下的层次结构: AS 级拓扑 在商业关系的约束下不存在 $\mathrm{c} 2 \mathrm{p}$ (客户 $\rightarrow$ 提供商) 的环路结构, 例如, 对于任意 3 个自治系统 $A, B$ 和 $C$, 若 $A$ 是 $B$ 的客户, $B$ 是 $C$ 的客户, 那么 $C$ 不可能是 $A$ 的客户, 否则 $A \rightarrow B \rightarrow C \rightarrow A$ 就形成了一个 迂回的环路, 从而造成数据包的循环传送. 这一约束严格定义了 Internet 的层次结构: 任何 AS 在拓 扑中的层次都在它所有的提供商之下. 实际的 Internet 存在少数 (10 20 个) 节点度数超大的 “顶级 
(Tier-1)”AS, 这些 AS 位于网络的最顶层, 它们彼此之间通过直连的对等连接 (p2p) 形成一个全互连 的子图 [7]. 顶级 AS 只为其他 AS 提供转发服务, 而不作为任何其他 AS 的客户, 它们各自拥有成百 上千的客户 AS. 在确定所有的顶级 AS 之后, 根据 AS 之间的商业关系可逐级推断出每一层次的 AS 集合, 从而确定所有 AS 所属的层次. 一般性地, 根据 AS 节点在层次结构中所处位置的不同, 我们把 AS 划分为 3 类: 顶级 AS (core AS, 没有提供商)、传输 AS (transit AS, 既有客户, 也有提供商) 和边 缘 AS (stub AS, 没有客户). 本文后续章节将用非顶级 AS 来代表传输 AS 和边缘 AS 的合集.

文献 [8] 利用 AS 商业关系提出了 AS 级拓扑的分层算法, 其算法大致分为两步: 1) 如果一个 AS 没有提供商 (即不存在以该 $\mathrm{AS}$ 为客户节点的 $\mathrm{c} 2 \mathrm{p}$ 连接), 那么该 $\mathrm{AS}$ 被视为一个顶级 $\mathrm{AS} ; 2$ ) 在确定了 所有顶级 $\mathrm{AS}$ 之后, 对于一个非顶级 $\mathrm{AS}$, 如果它有一个提供商 $\mathrm{AS}$ 在第 $i$ 层, 那么该 $\mathrm{AS}$ 则被划分到第 $i+1$ 层 (该 $\mathrm{AS}$ 的提供商都分布在 $i+1$ 层之上). 基于以上层次划分, 可根据关系类型图 $G=(V, E, R)$ 得到与之对应的层次关系类型图 $G_{H}=(V, E, R, H)$, 其中, $H$ 是 $\mathrm{AS}$ 节点到层次序号的映射. 比如, 对 于一个第 $m$ 层的 AS 节点 $i$, 它所对应的 $H$ 值为 $H(i)=m$. 直观地, 我们用 $V_{m}$ 表示第 $m$ 层的节点 集合, 那么, 顶级节点集合就可表示为 $V_{1}$.

\section{3 单个 $\mathrm{AS}$ 的健壮性}

$\mathrm{AS}$ 级拓扑的层次特性决定了非顶级 $\mathrm{AS}$ 的大部分流量需要依靠其上游的 AS 来转发. 因此, 大多 数 AS 都是通过简单的链路备份或 “多宿主” 来增强其健壮性. 本节将深入展开对单个 AS 健壮性的 研究, 探讨对单个 AS 的健壮性测度与健壮性演化.

\section{1 单个 $\mathrm{AS}$ 的健壮性测度}

正常状态下, Internet 的任意两个 AS 节点都相互可达, 即对于任意两个给定的 AS, 它们之间必 然存在一条符合 “无谷底” 约束的 AS 路径. 否则, 分属于这两个自治系统的主机相互之间就不能建立 网络连接. 就单个 AS 拓扑的连通性而言, 我们将论述两点: 1) 对于顶级 AS 之间的连通性, 需要维持 顶级 AS 的全互连子图; 2) 而对于每个非顶级 AS, 它的连通性取决于它所拥有的到达最上游 (顶级) $\mathrm{AS}$ 的不相交上坡路径数.

推断 1 (顶级 AS 的全互连结构) 任意两个顶级 AS 之间都存在直连的对等连接 (p2p), 彼此之 间形成了一个全互连的子图.

证明 对于任意两个给定的顶级 $\mathrm{AS} A$ 和 $B, A$ 和 $B$ 相互可达, 如果它们之间没有一条直连的 $\mathrm{p} 2 \mathrm{p}$ 连接, 假设通过第三个 $\mathrm{AS}$ 节点 $C$ 实现它们之间的可达性, 即 $\mathrm{A} \leftrightarrow C \leftrightarrow \mathrm{B}$. 由于 $A$ 是顶级 $\mathrm{AS}, C$ 只 可能是 $A$ 的客户或对等 $\mathrm{AS}$; 同样地, 由于 $B$ 也是顶级 $\mathrm{AS}, C$ 只可能是 $B$ 的客户或对等 AS. 1) 如果 $C$ 是 $A$ 的客户 $\mathrm{AS}$, 由于 $B$ 是顶级 $\mathrm{AS}, C-B$ 只能是一条 $\mathrm{p} 2 \mathrm{p}$ 连接或 $\mathrm{c} 2 \mathrm{p}$ 连接, 显然, 这两种情况都 违背了 “无谷底” 约束, 因此 $A$ 和 $B$ 不可达; 2) 如果 $C$ 是 $A$ 的对等 AS, 无论 $C-B$ 是 p2p 连接还 是 $\mathrm{c} 2 \mathrm{p}$ 连接, 这两种情况都违背了 “无谷底” 约束, 因此 $A$ 和 $B$ 不可达. 因此, 正常情况下, 任意两个 顶级 AS 要想保持相互的可达性, 它们之间就必定存在一条 $\mathrm{p} 2 \mathrm{p}$ 直连边, 所有的顶级 AS 以及它们之 间的 $\mathrm{p} 2 \mathrm{p}$ 直连边也就形成了一个全互连的子图.

在实际的 Internet 中, 绝大多数的 ISP 依赖于它们上游的 ISP 提供转发服务. 切断一个 AS 的所 有上游链路也就使得该 AS 和 Internet 的主体分割开来. 因此, 大多数 ISP 都通过简单的备份上游链 路或者以多宿主的方式来增强其健壮性. 然而，单纯地依靠这些方式还不足以确保一个 AS 的健壮性 


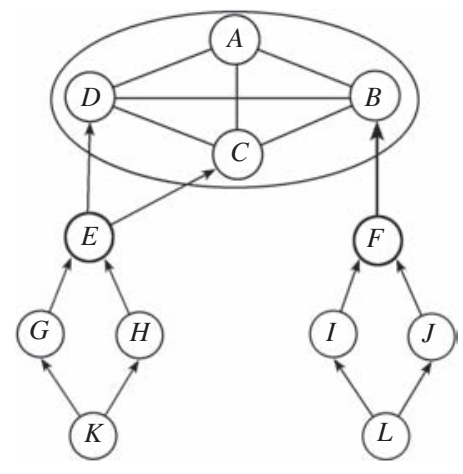

图 1 多个上游链路与单故障切断

Figure 1 ASes with multiple upstream links disconnected by single failures

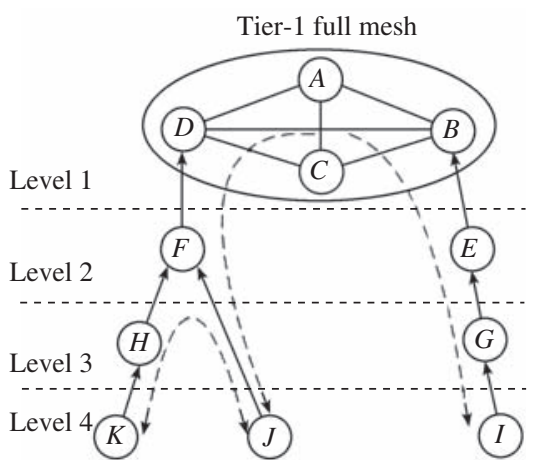

图 2 拥有顶级上坡路径的 AS 之间的连通性

Figure 2 Reachability between AS pairs with Tier-1 uphill paths

得以增强. 即便一个 AS 拥有多条上游链路, 它仍有可能在单个上游链路/节点发生故障的情况下失去 到上游的连通性. 例如, 如图 1 所示, 尽管节点 $K$ 和 $L$ 都有两条上游链路, 单个节点故障 (节点 $E$ ) 就 足以把节点 $K$ 到上游的连通性切断; 而对于节点 $L$, 单个节点故障 (节点 $F$ ) 或单个链路故障 $F \rightarrow B$ 都可以切断它到上游的连通性. 进一步考虑单个 AS 的连通性测度. 为此, 首先引入以下定义.

定义 2 (顶级上坡路径, Tier-1 uphill path) 对于任意给定的一个非顶级 AS, 记为 $n_{0}$, 若存在一 条上坡路径 $n_{0} \rightarrow n_{1} \rightarrow \cdots \rightarrow n_{i} \rightarrow n_{i+1} \rightarrow \cdots \rightarrow n_{j}$, 其中 $n_{i} \rightarrow n_{i+1}(0 \leqslant i \leqslant j-1)$ 是一条 $\mathrm{c} 2 \mathrm{p}$ 连接, $H\left(n_{j}\right)=1$ 且 $n_{j}$ 是该路径中唯一的顶级 $\mathrm{AS}$, 那么, 我们称该上坡路径为 $n_{0}$ 的一条顶级上坡路径.

对于每个非顶级 AS, 它的顶级上坡路径代表了一条从该 AS 到达一个顶级 AS 的上坡路径. 正常 情况下, 每个非顶级 $\mathrm{AS}$ 都至少存在一个上游服务提供商. 由此可推断, 每个非顶级 $\mathrm{AS}$ 至少拥有一条 顶级上坡路径. 例如, 对于一个处于第 $m$ 层的 $\mathrm{AS}(A)$, 由于它是非顶级 $\mathrm{AS}$, 它必然能通过一条 $\mathrm{c} 2 \mathrm{p}$ 的 连接到达一个上游 (更高层) 的 $\mathrm{AS}(B)$, 如果 $B$ 是顶级 $\mathrm{AS}$, 那么 $A-B$ 就是 $A$ 的一条顶级上坡路径; 如果 $B$ 是非顶级 $\mathrm{AS}$, 那么 $B$ 必然能通过一条 $\mathrm{c} 2 \mathrm{p}$ 的连接到达一个更高层次的 $\operatorname{AS}(C)$, 依次类推, $A$ 总能找到一条到达顶级 AS 的上坡路径.

推断 2 (任意两个拥有顶级上坡路径的 AS 必然相互可达) 对于任意两个非顶级 AS, 如果它们 各自至少拥有一条顶级上坡路径, 那么它们之间必然存在一条满足 “无谷底” 约束的 AS 路径, 使得它 们之间相互可达.

证明 假设两个非顶级 AS 各自拥有一条顶级上坡路径, 这两条路径的关系无非相交或者不相交 两种情况.

1) 如果两条路径相交, 如图 2 的节点 $K$ 和 $J$, 它们各自的上坡路径为 $K-H-F-D$ 和 $J-F-D$, 两条路径的节点交集为 $\{F, D\}$. 取交集中层值最大的 AS 节点 (节点 $F, H(F)=2>H(D)=1$ ), 则从 $K$ 到 $F$ 是一条上坡路径, 从 $F$ 到 $J$ 是一条下坡路径, 根据路径模式 $5, K$ 和 $J$ 之间存在一条 “上坡 路径 +下坡路径” 模式的路径 $K-H-F-J$, 因此, $K$ 和 $J$ 可达.

2 ) 如果两条路径不相交, 如图 2 的节点 $J$ 和 $I$, 由于它们各自分别拥有一条顶级上坡路径 $J-F-D$ 和 $I-G-E-B$ 分别到达两个不同的顶级 $\mathrm{AS}(D$ 和 $B)$, 根据推断 $1, D$ 和 $B$ 之间存在一条 $\mathrm{p} 2 \mathrm{p}$ 直 连边, 根据模式 $6, K$ 和 $I$ 之间必然存在一条 “上坡路径 + 一条 $\mathrm{p} 2 \mathrm{p}$ 连接 + 下坡路径” 模式的路径 $J-F-D-B-E-G-I$, 因此, $J$ 和 $I$ 可达.

综上, 任意两个拥有顶级上坡路径的 AS 必然相互可达.

根据推断 2 , 对于非顶级 AS 来说, 拥有顶级上坡路径 (而非多少条上游备份链路) 是其拥有全网 


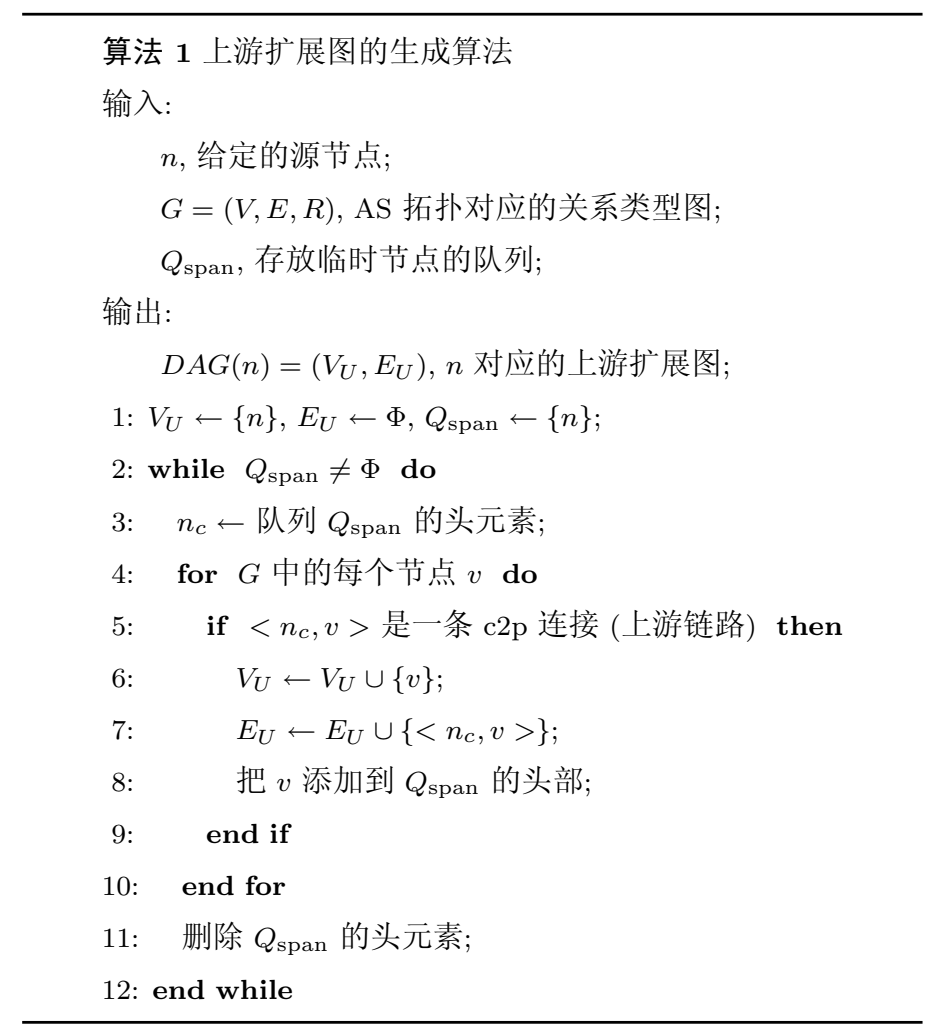

连通性的必要条件. 一旦失去所有的顶级上坡路径, 也就意味着该节点与最上游的网络节点失去了连 通性. 因此, 非顶级 AS 的健壮性取决于它的顶级上坡路径应对节点/链路失效的能力. 在经典的图 论里面, 两个节点对之间的连通健壮性常用最小节点/边割集 (minimum vertex/edge cut) 来度量. 根

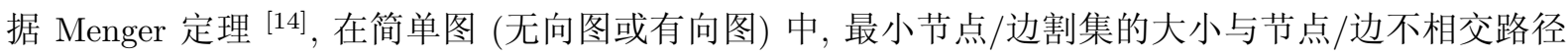
数 (node-/edge-disjoint path) 是相等的. 把 $\mathrm{c} 2 \mathrm{p}$ 连接看作是从客户到提供商的有向边, 由于 “客户 $\rightarrow$ 提供商” 关系的无环约束 ${ }^{[13]}$, 从节点 $n$ 出发的所有上坡路径所覆盖的所有节点与 $\mathrm{c} 2 \mathrm{p}$ 连接构成了一 个有向无环图 (directed acyclic graph, DAG), 记为 $\operatorname{DAG}(n)$, 我们称之为节点 $n$ 对应的上游扩展图. 对 于一个非顶级节点 (AS n), 如果它拥有 $k$ 条节点/边不相交的顶级上坡路径 ${ }^{3)}$, 那么, 任意 $(k-1)$ 个 节点/链路失效都不会导致它与上游 AS 的连通性被切断. 因此, 单个 AS 节点对节点/链路故障的健 壮性取决于以下两个指标.

定义 3 (边不相交的顶级上坡路径数, $\left.N_{\mathrm{edp}}\right) \quad N_{\mathrm{edp}}(n)$ 定义为节点 $n$ 的边不相交的顶级上坡路 径数.

定义 4 (节点不相交的顶级上坡路径数, $N_{\mathrm{ndp}}$ ) $\quad N_{\mathrm{ndp}}(n)$ 定义为节点 $n$ 的节点不相交的顶级上 坡路径数.

为了计算单个 $\operatorname{AS}(\operatorname{AS} n)$ 所拥有的节点/边不相交的顶级上坡路径数, 首先需要从关系类型图 $G$ 中 提取出该 AS 所对应的上游扩展图 (DAG(n)) (如算法 1 所示). 图 3 给出了从关系类型图 $G=(V, E, R)$ 中提取 DAG 的例子. 在得到单个 AS 节点的 DAG 之后, 不相交路径数的计算又可等价转化为单元容 量网络 (unit capacity network, 即每个边的容量为单位 1 的流网络) 的最大流问题 ${ }^{[15,16]}$, 进一步可计

3) 在这里, 两条节点不相交的顶级上坡路径是指这两条路径除了起始 AS 节点之外, 没有其他交叉点, 即没有其他 共有的 AS 节点; 两条边不相交的顶级上坡路径是指这两条路径没有共有的链路 


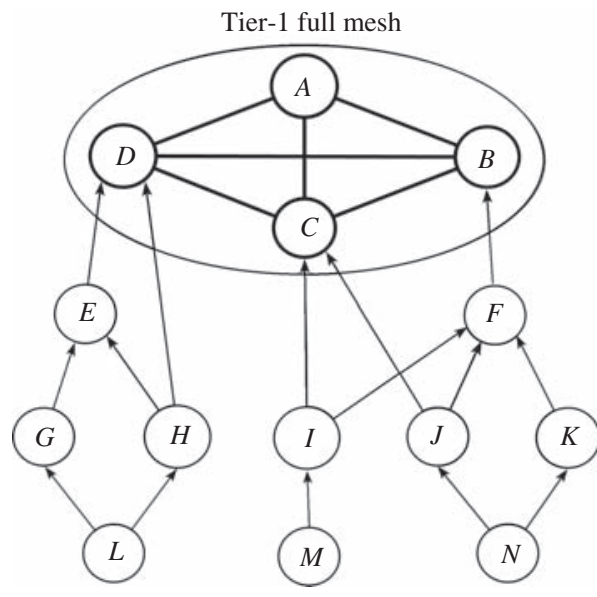

(a)

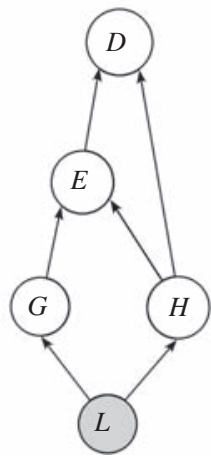

(b)

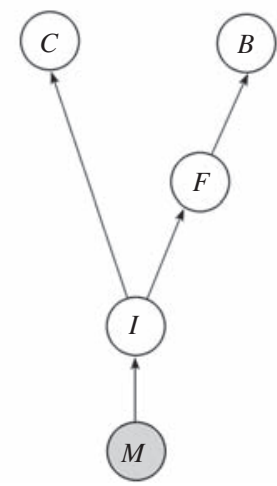

(c)

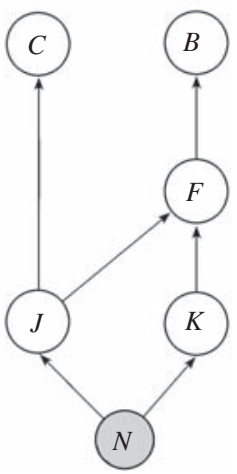

(d)

图 3 DAG 的提取实例

Figure 3 Examples for DAG construction. (a) The given ToR graph G; (b) DAG $(L)$; (c) DAG(M); (d) DAG $(N)$

算出它所对应的 $N_{\mathrm{edp}}$ 与 $N_{\mathrm{ndp}}$. 如图 3, 节点 $L$ 对应的 $N_{\mathrm{edp}}(L)=2, N_{\mathrm{ndp}}(L)=1$, 因此, 在任何一个链路 失效的情况下, 节点 $L$ 都能够它到上游的连通性, 而当节点 $D$ 失效时, 节点 $L$ 就失去了它的所有顶 级上坡路径. 节点 $M$ 对应的 $N_{\text {edp }}(M)=1$ 且 $N_{\text {ndp }}(M)=1$; 节点 $N$ 对应的 $N_{\text {edp }}(N)=2$ 且 $N_{\text {ndp }}(N)=2$. 相比来说, 节点 $N$ 的连通性比节点 $M$ 和 $L$ 都要健壮.

\section{2 单个 AS 的健壮性演化}

如图 1, 我们论述了拥有多条上游备份链路的 AS 并不能保证它对单个故障的健壮性, 即拥有多 个上游链路并不代表拥有多条不相交的顶级上坡路径. 因此, 对于任何一个非顶级 AS, 为它增加一条 新的上游备份链路并不意味着能增加一条新的顶级上坡路径, 这也就限制了当今广泛采用的链路备份 及多宿主技术增强单个节点健壮性的能力. 给定一个 AS (AS n), 假设它拥有 $k$ 条边/节点不相交的顶 级上坡路径, 因此, 在 $k$ 个链路/节点同时发生故障的情况下, AS $n$ 可能会失去所有的顶级上坡路径. 为了能通过增加一条上游备份链路的方式来为 $\operatorname{AS} n$ 增加一条不相交的顶级上坡路径, 我们引入可选 集的概念.

定义 5 (可选集, $\left.V_{e}(n)\right) \quad V_{e}(n)$ 是一个 $\mathrm{AS}$ 节点集合, 对于任意 $v \in V_{e}(n), v$ 都满足以下两个条 件: 1) $H(v)<H(n)$, 即 AS $v$ 处于 AS $n$ 的上游, 并且 2) 从 $v$ 到 $n$ 增加一条新的 $\mathrm{c} 2 \mathrm{p}$ 连接 $n \rightarrow v$ 可 以使得 $\mathrm{AS} n$ 所拥有的边/节点不相交的顶级上坡路径在原有的基础上增加 1, 我们称之为节点 $n$ 的 边/节点不相交可选集.

基于可选集的定义, 单个 $\mathrm{AS}$ 健壮性增强的启发式策略可概括为: 1) 对于给定的关系类型图 $G$ 和 当前 $\mathrm{AS}(\mathrm{AS} n)$, 计算出 $\mathrm{AS} n$ 的可选集 $\left.V_{e}(n) ; 2\right)$ 从 $\mathrm{AS} n$ 的可选集 $V_{e}(n)$ 中任意选出一个 $\mathrm{AS}(\mathrm{AS}$ $v)$, 增加一条从 $\operatorname{AS} n$ 到 $\mathrm{AS} v$ 的上游备份链路 $\langle n, v\rangle$. 图 4 分别给出了增加边不相交顶级上坡路径 $V_{e}(M)$ 和增加节点不相交顶级上坡路径的可选集 $V_{e}^{\prime}(M)$, 其中, 节点 $M$ 是当前 $\mathrm{AS}$, 边不相交可选集 为 $V_{e}(M)=\{A, B, C, D, E, F, G, H, J, K\}$, 节点不相交可选集为 $V_{e}^{\prime}(M)=\{A, B, D, E, F, G, H, K\}$. 特 别的, 增加一条从 $M$ 到 $J$ 的 $\mathrm{c} 2 \mathrm{p}$ 链路能够为 $\mathrm{M}$ 增加一条新的边不相交顶级上坡路径, 但是并不能为 $M$ 增加一条新的节点不相交顶级上坡路径，因此，节点 $J$ 属于节点 $M$ 的边不相交所对应的可选集， 


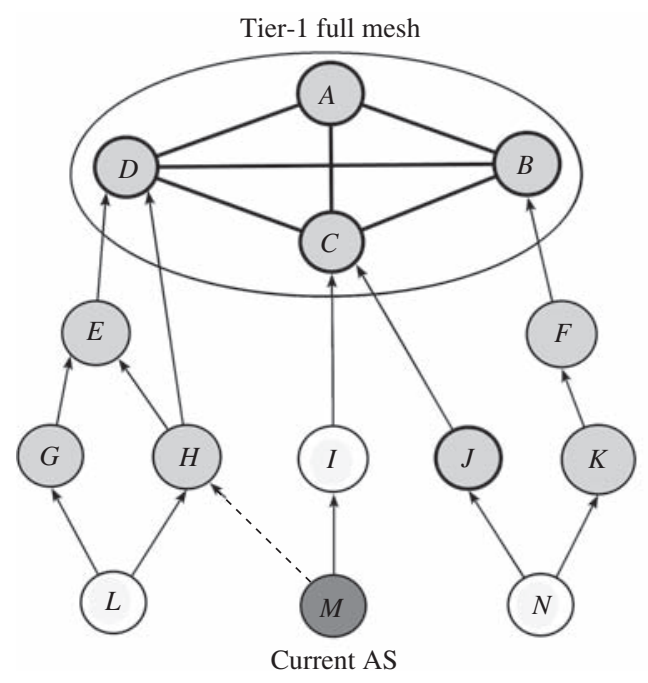

(a)

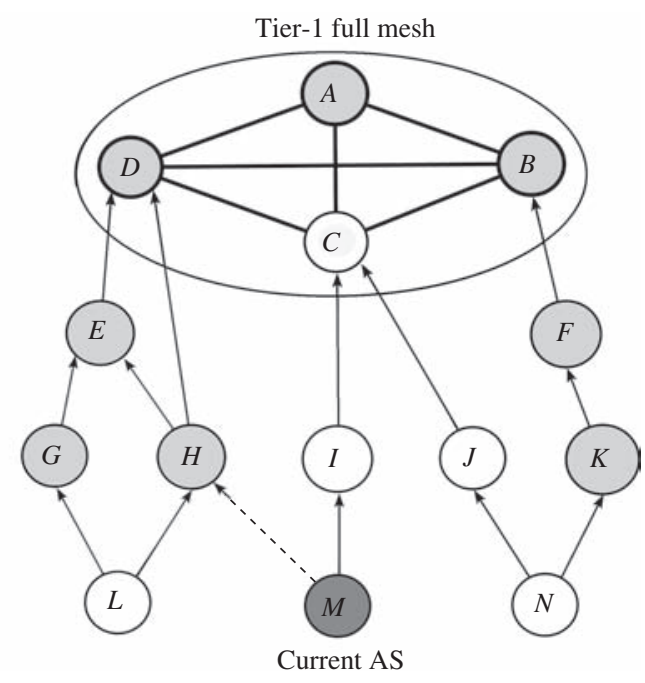

(b)

图 4 为当前节点 $(M)$ 增加一条边/节点不相交顶级上坡路径的可选集

Figure 4 Candidate set for adding one edge-/node-disjoint Tier-1 uphill paths for current node ( $M)$. (a) Candidate set for edge-disjoint; (b) candidate set for node-disjoint

而不属于其节点不相交所对应的可选集.

\section{4 全局 $\mathrm{AS}$ 级拓扑的 $k$ 容错}

$k$ 容错的概念被广泛用于研究各种网络拓扑的健壮性, 从全局上刻画了一个网络拓扑的容错能力 与鲁棒性. 从简单图出发, 它的基本意义是在任意 $k$ 个节点/边被移除的情况下, 剩余网络的各个节点 对之间仍然存在连通性. 而 Internet AS 级拓扑由于商业关系以及对 AS 路径的 “无谷底” 约束的存 在, 使得 AS 级拓扑对应的关系类型图远远超出了简单图的范畴. 因此, AS 级拓扑所对应的 $k$ 容错也 相应变得复杂.

\subsection{AS 级拓扑的 $k$ 容错判定}

由于 $\mathrm{AS}$ 级拓扑所对应的关系类型图与简单图有着显著的差别, 我们首先给出 $\mathrm{AS}$ 级拓扑的 $k$ 容 错定义.

定义 6 (AS 级拓扑的 $k$ 容错) 对于一个给定的关系类型图 $G$ ( $G$ 对应于 Internet 的 AS 及拓扑), $G$ 是 $k$ 容错的当且仅当在任意节点被移除的情况下, 剩余网络中的任意两个节点之间仍然相互可达, 即任意两个节点之间仍然存在一条满足 “无谷底”约束的 AS 路径.

文献 $[17,18]$ 指出, 计算关系类型图的节点对之间的最小割集是一个 NP 难题. 对于一个给定的关 系类型图 $G$, 如果以穷举的方式判定其 $k$ 容错, 最坏的情况下需要遍历每个节点对, 计算每个节点对 之间的最小割集是否都大于 $k$. 显然, 在这种方式下的 $k$ 容错判定也是 NP 难的. 为了更有效地判定 $\mathrm{AS}$ 级拓扑的 $k$ 容错, 我们从 $\mathrm{AS}$ 级拓扑的层次结构特性出发, 推断出 AS 级拓扑 $k$ 容错的充分必要 条件.

定理 1 (AS 级拓扑 $k$ 容错的充要条件) ${ }^{[19]}$ 对于一个给定的关系类型图 $G=(V, E, R)$, 它对应 的层次化关系类型图为 $G_{H}=(V, E, R, H) . G$ 是 $k$ 容错的当且仅当 $G$ 同时满足以下两个条件: 
1) 顶级 $\mathrm{AS}$ 之间的全互连: 所有的顶级 $\mathrm{AS}\left(V_{1}\right)$ 之间都存在一条直连的对等关系 (p2p) 连接, 即 顶级 AS 之间通过 $\mathrm{p} 2 \mathrm{p}$ 连接形成全互连结构;

2) 每个非顶级 $\mathrm{AS}$ 拥有 $>k$ 条上游链路: 所有的非顶级 $\mathrm{AS}\left(V \backslash V_{1}\right)$ 都有 $>k$ 条 $\mathrm{c} 2 \mathrm{p}$ 连接到不同 的上游 $\mathrm{AS}$, 即有 $>k$ 个提供商 $\mathrm{AS}$.

证明 对该定理的证明包括充分性和必要行的证明.

充分性证明 考虑有 $k$ 个节点同时失效的情况下, 记为 $F=\left\{n_{1}, n_{2}, \ldots, n_{k}\right\}$. 由于每个非顶级 $\mathrm{AS}$ 都有 $>k$ 条上游链路, 因此, 剩余网络中的任意非顶级 $\mathrm{AS}(v)$ 都至少还有一条上游连接到达一个 上游 $\mathrm{AS}\left(v^{\prime}\right)$. 如果 $v^{\prime}$ 是顶级 $\mathrm{AS}$, 那么 $v-v^{\prime}$ 就是 $v$ 的一条顶级上坡路径; 如果 $v^{\prime}$ 是非顶级 $\mathrm{AS}$, 那 么 $v^{\prime}$ 必然能通过一条 $\mathrm{c} 2 \mathrm{p}$ 的连接到达一个更高层次的 $\operatorname{AS}\left(v^{\prime \prime}\right)$, 依次类推, $v$ 总能找到一条达到顶级 AS 的上坡路径. 由于顶级 AS 之间是 p2p 全互连的, 由 “无谷底” 特性和推断 2 知, 任意顶级 AS 之 间 (p2p 直连)，顶级 AS 和非顶 AS 之间 (“上坡路径”, 或者 “上坡路径 +一条 p2p 连接”)，以及非顶 级 AS 和非顶级 AS 之间 (“上坡路径 + 一条 $\mathrm{p} 2 \mathrm{p}$ 连接”, 或者 “上坡路径 + 一条 $\mathrm{p} 2 \mathrm{p}$ 连接 + 下坡路 径”) 都存在有效的路径, 因此, 剩余网络中的任意节点对之间仍然相互可达.

必要性证明 条件 1) 的必要性可由推断 1 直接得证. 下面证明条件 2) 的必要性, 假设存在一个 非顶级节点 $v$ 只有 $k$ 个上游提供商, 如果有 $k$ 个节点同时失效, 最坏的情况下, $v$ 的所有上游提供商 AS 都失效, 那么 $v$ 将失去所有的顶级上坡路径, 因此, $v$ 和它在剩余网络中的上游节点之间的连通性 将被切断.

由此, 定理 1 描述了 Internet AS 级拓扑基本的 $k$ 容错模型. 由于该模型主要刻画了 AS 级拓扑 对节点失效的 $k$ 容错, 接下来讨论该模型对 $\mathrm{AS}$ 级链路失效的容错能力. 推断 1 论述了两个顶级 AS 之间的可达性的必要条件是它们之间存在直连的 $\mathrm{p} 2 \mathrm{p}$ 连接, 两个顶级 AS 之间的路径只有一条, 因此, 顶级 AS 之间的连通性永远是 0 容错的. 然而, 文献 [20] 的研究结果表明, 大多数顶级 AS 之间的 p2p 连接都存在多条物理级的备份链路, 即两个顶级 AS 之间的逻辑上的 $\mathrm{p} 2 \mathrm{p}$ 连接通常包含多条物理链路. 如果不考虑顶级 AS 之间的 $\mathrm{p} 2 \mathrm{p}$ 链路失效, 定理 1 所刻画的 $k$ 容错模型对链路失效同样是成立的, 即 在任意 $k$ 个链路 (不包含顶级 AS 之间的 $\mathrm{p} 2 \mathrm{p}$ 连接) 失效的情况下, 任意两个节点之间仍然相互可达.

根据定理 1 所描述的两个必要条件, 对 AS 级拓扑的 $k$ 容错判定简化为以下两个步骤: 1) 检查顶 级 $\mathrm{AS}$ 是否通过 $\mathrm{p} 2 \mathrm{p}$ 连接形成全互连结构; 2) 检查每个非顶级 AS 是否有大于 $k$ 个提供商 AS. 由于 顶级 AS 的数量只有 10 20, 因此, 第 1 步的开销可认为是常数时间内的; 而第 2 步需要遍历每个非 顶级 AS 并计算它们的上游连接数, 时间开销为 $O(|V|+|E|)$. 因此, AS 级拓扑 $k$ 容错判定的时间复 杂度为 $O(|V|+|E|)$.

\subsection{AS 级拓扑的 $k$ 容错演化}

根据定理 1 , 进一步阐述怎样基于现有的 AS 级拓扑以最小的代价将其演化成一个 $k$ 容错的拓扑. 由于顶级全互连是 Internet AS 级拓扑的一个基本假设 ${ }^{[20]}$, 着重考虑达到 AS 级拓扑 $k$ 容错的第二 个条件所需要增加的链路数. 遍历所有的非顶级 AS 节点, 如果存在一个 AS, 假设它的上游链路数为 $m$, 若 $m<(k+1)$, 则为它增加 $(k+1-m)$ 条额外的上游备份链路到它的上游 AS. 由此, 我们给出 $k$ 容错演化的代价估算如下:

$$
\left|E_{\text {extra }}\right|=\sum_{i=0}^{k}(k+1-i) \cdot N_{i},
$$




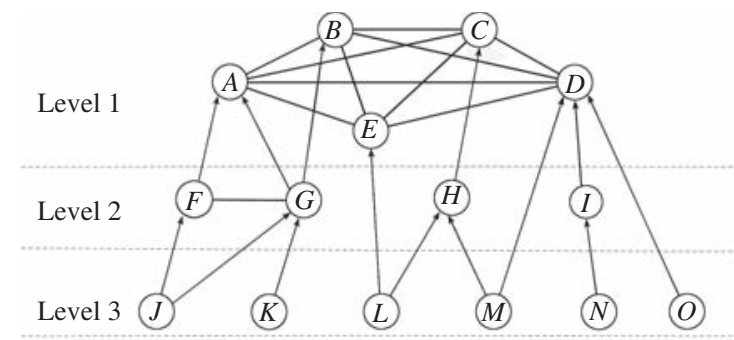

(a)

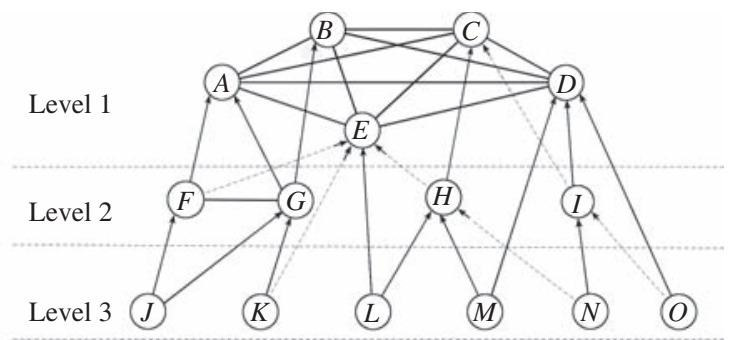

(b)

图 5 -个 1 容错的演化实例

Figure 5 An example for 1-fault tolerance augmentation. (a) The original AS topology; (b) the 1-fault tolerant topology

其中, $E_{\text {extra }}$ 是需要新增的上游链路集合, $N_{i}$ 是拥有 $i(i=0,1, \ldots, \mathrm{k})$ 条上游链路的 AS 数, 对于这类 $\mathrm{AS}$ 节点, 需要额外的 $(k+1-i) \cdot N_{i}$ 条上游备份链路使它们满足定理 1 的条件 2). 因此, AS 拓扑的 $k$ 容 错演化的具体步骤分为以下两步:

1) 如果顶级 AS 之间不是一个以 $\mathrm{p} 2 \mathrm{p}$ 连接形成的全互联结构, 在那些没有 $\mathrm{p} 2 \mathrm{p}$ 连接的顶级 AS 之 间增加额外的 $\mathrm{p} 2 \mathrm{p}$ 连接, 使得顶级 AS 之间形成一个 p2p 的全互联结构;

2) 对于非顶级 AS 节点, 如果它的上游连接数少于 $k+1$, 那么, 从它上层的 AS 中选择新的 AS 作 为它的提供商, 增加相应的上游连接, 使得其上游连接数达到 $k+1$.

图 5 给出了将一个给定的关系类型图演化为一个 1 容错拓扑的例子, 其中, 在初始的关系类型图 中, 存在 6 个非顶级 AS, 它们的上游链路数小于 $(k+1)$ 且均为 $1(m=1)$, 因此, 将图 5(a) 所对应的拓 扑演化为 1 容错的最小代价 $\left|E_{\text {extra }}\right|=6$. 图 5(b) 给出的 1 容错实例所对应的 $E_{\text {extra }}=\{\langle F, E\rangle,\langle H, E\rangle$, $\langle I, C\rangle,\langle K, E\rangle,\langle N, H\rangle,\langle O, I\rangle\}$. 需要指出的是, 理论上, AS 可以任意地选取一个上游 AS 来增加一条 新的上游链路, 但在实际中, 运营商并没有这样的自由, 增加一条新的上游链路意味着高昂的成本, 并 且需要运营商的双边达成商业协议, 建立 AS 之间的商业关系.

\section{5 实验分析}

第 3 节和第 4 节分别从 “单个 AS” 和 “全局 AS 级拓扑” 两个不同层面对 Internet AS 级拓扑的 健壮性测度与健壮性进行了深入的研究. 本节将结合实际的 AS 级拓扑数据, 采用前面的指标与模型, 评估 Internet AS 级拓扑的健壮性, 并进一步探讨本文所提出的测度方法与演化机制对 Internet 健壮 性的实际意义.

\subsection{AS 级拓扑的数据源及基本统计}

由于现有的各种 AS 级拓扑数据源都不能反映 Internet 的完整视图 ${ }^{[21]}$, 本文通过融合多个 AS 级 拓扑数据源以尽可能减小数据的不完整性对实验评估的影响: CAIDA 在 2009 年 8 月 30 日的数据集 和 AquaLab 在 2009 年 8 月 28 日的数据集, 其中, CAIDA 的数据集是根据 RouteViews ${ }^{4}$ 采集的 BGP 路由表推断而来的; AquaLab 通过分布式的 P2P 系统对 BGP 路径进行 Traceroute 之后再进一步推 断得到 AS 商业关系数据集 ${ }^{222]}$. 如表 1 所示, CAIDA 的数据源观包含的 AS 数目较多 (32381 个 AS), 而 AquaLab 的连接数更多, 其中, p2c(c2p) 连接占了大多数. 合并后得到的 AS 级拓扑包括 35273 个 AS、 115768 条 $\mathrm{p} 2 \mathrm{c}(\mathrm{c} 2 \mathrm{p})$ 连接和 51432 条 $\mathrm{p} 2 \mathrm{p}$ 连接.

4) Http://www.routeviews.org 
表 1 AS 级拓扑数据来源

Table 1 Data sources for AS topology

\begin{tabular}{cccc}
\hline \hline Data sources & Number of ASes & \multicolumn{2}{c}{ Number of AS relationships } \\
\cline { 3 - 4 } & & $\mathrm{p} 2 \mathrm{c}$ & $\mathrm{p} 2 \mathrm{p}$ \\
\hline CAIDA & 32,381 & 66,498 & 6,321 \\
AquaLab & 31,554 & 93,620 & 48,216 \\
Merged topology & 35,273 & 115,768 & 51,432 \\
\hline
\end{tabular}

表 2 非顶级 $\mathrm{AS}$ 的上游链路数统计

Table 2 Statistics for upstream links of non-Tier-1 ASes

\begin{tabular}{cc}
\hline \hline$m$ & Number of non-Tier-1 ASes with $m$ upstream links $(\# / 35259)$ \\
\hline 0 & $19(0.05 \%)$ \\
1 & $7709(21.86 \%)$ \\
2 & $13074(37.08 \%)$ \\
4 & $6384(18.1 \%)$ \\
5 & $3057(8.67 \%)$ \\
$>5$ & $1579(4.48 \%)$ \\
\hline
\end{tabular}

由于并不存在一个权威公认的顶级 AS 列表, 需要从 AS 级拓扑推断出顶级 AS. 受 AS 级拓扑视 图不完整性的限制 [21], 采用文献 [8] 的方法所推断出来的顶级 AS 可能存在明显的误差 (由于实际采 集到的拓扑可能不完整, 一个 AS 没有上游提供商并不代表它一定是顶级 AS). 为此, 我们对顶级 AS 的推断作以下改进: 1) 如果一个给定的 $\mathrm{AS}$ 节点度数超过一个给定的阈值 $D(D=600)$, 则把该节点 列入候选的顶级节点; 2) 遍历完所有的节点之后, 得到所有的候选顶级节点集; 3) 对于候选集的每一 个 $\mathrm{AS}$, 如果该 $\mathrm{AS}$ 和候选集里 $P \%$ (比如, $80 \%$ ) 的节点存在 $\mathrm{p} 2 \mathrm{p}$ 连接,则把该节点划分到顶级 $\mathrm{AS}$ 集. 得到了 14 个顶级 AS, 包括 AS 174 (Cogent), 209 (Quest), 701 (UUnet), 1239 (Sprintlink), 2828 (XO Com.), 2914 (NTT-Com.), 3356 (Level3), 3549 (Gblx), 7018 (AT\&T WorldNet) 等, 和 Wikipedia ${ }^{5)}$ 以 及 $\mathrm{CAIDA}^{6}$ ) 所列出的顶级 $\mathrm{AS}$ 基本一致.

除去 14 个顶级 AS, 剩余 35259 个非顶级 AS. 表 2 给出了非顶级 AS 的上游链路数统计, 其中, 19 个非顶级 AS 没有上游链路 (因为拓扑视图的不完整性), 即 $N_{0}=19 ; 7709$ 个 AS 只有一条上游 链路, 即 $N_{1}=7709$; 大约 $78 \%$ 的非顶级 AS 拥有多个提供商. 由于存在大约 $22 \%$ 的非顶级 AS 只 有一个上游提供商 $\mathrm{AS}$, 根据定理 1 可判定, 给定的 $\mathrm{AS}$ 级拓扑实例是 0 容错的. 根据式 (1) 知, 将其 演化为 1 容错代价为: $\left|E_{\text {extra }}\right|=\sum_{i=0}^{1}(1+1-i) \cdot N_{i}=19 \times 2+7709=7447$; 将其演化为 2 容错的代价 为: $\left|E_{\text {extra }}\right|=\sum_{i=0}^{2}(2+1-i) \cdot N_{i}=19 \times 3+7709 \times 2+13074=28549$.

\section{2 单个 $\mathrm{AS}$ 的健壮性}

根据第 3 节的论述, 单个 AS 的健壮性并非取决于它所拥有的上游链路数, 而是取决于它所拥有 的边/节点不相交的顶级上坡路径数. 一个 AS 拥有多条边/节点不相交的顶级上坡路径意味着该节点

5) Http://en.wikipedia.org/wiki/Tier_1_network

6) Http://www.caida.org/research/topology/rank_as/ 


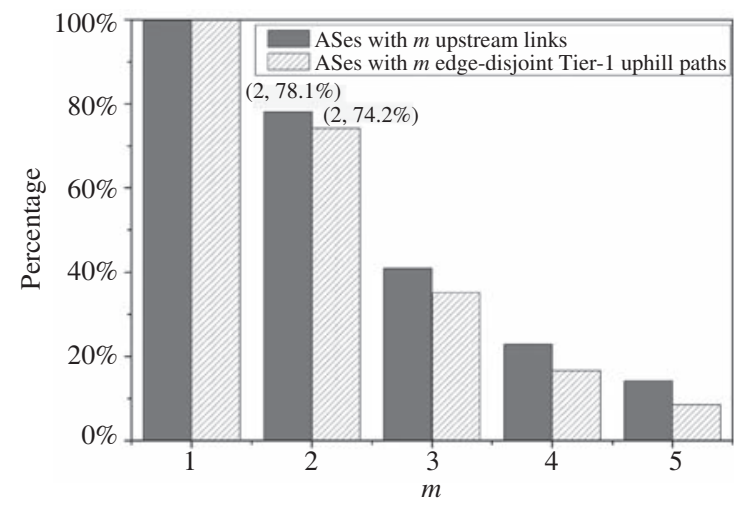

(a)

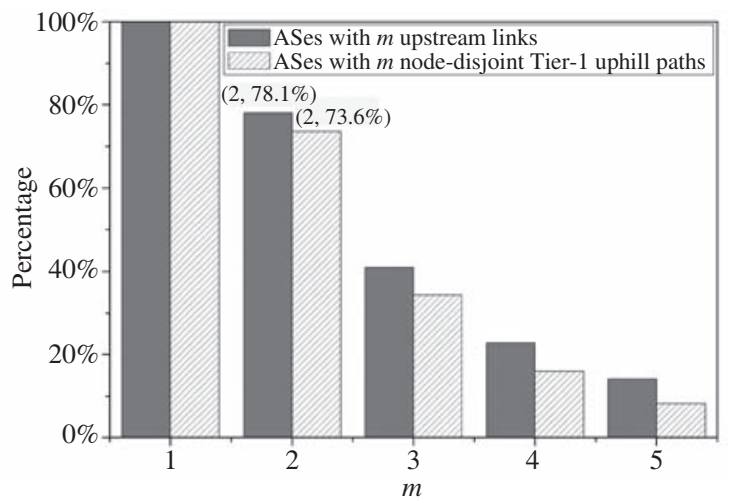

(b)

图 6 上游链路数 vs. 不相交的顶级上坡路径数

Figure 6 Number of upstream links vs. number of disjoint Tier-1 uphill paths. (a) Number of upstream links vs. number of edge-disjoint Tier-1 uphill paths; (b) number of upstream links vs. number of node-disjoint Tier-1 uphill paths

在任意一条链路/节点发生故障时仍然能通过它的上游和大多数其他网络节点保持连通性. 图 6 给出 了非顶级 AS 所拥有的不相交顶级上坡路径数统计, 其中, 图 6(a) 给出了 AS 拥有的上游链路数与 AS 拥有的边不相交顶级上坡路径数的比较, 图 6(b) 给出了 AS 拥有的上游链路数与 AS 拥有的节点不相 交顶级上坡路径数的比较.

不难发现, 对任意的非顶级 AS 来说, 拥有多条上游链路并不能保证它拥有多条不相交的顶级上 坡路径. 如图 6 所示, 尽管 $78.1 \%$ 的非顶级 AS 拥有 2 条以上的上游链路, 但是, 真正拥有 2 条以上的 边不相交顶级上坡路径的非顶级 $\mathrm{AS}$ 却只有 $74.2 \%$; 拥有 2 条以上的节点不相交顶级上坡路径的非顶 级 AS 更低—只有 73.6\%. 由此表明, 增加一条上游备份链路并不能保证 AS 的健壮性得以相应地 增强. 另外, 节点不相交的顶级上坡路径数略小于边不相交的顶级上坡路径数, 即一个 AS 拥有多条 边不相交的顶级上坡路径并不意味着它也拥有同等数量的节点不相交顶级上坡路径.

\section{$5.3 k$ 容错拓扑的健壮性}

根据 $\mathrm{AS}$ 级拓扑 $k$ 容错的定义知，任意 $k$ 个故障都不会破坏一个 $k$ 容错的 $\mathrm{AS}$ 级拓扑中节点对 之间的连通性. 那么, 当有超过 $k$ 个故障发生时, 与原始的 AS 级拓扑相比, $k$ 容错拓扑的健壮性又会 怎么样呢? 考虑到基于现有的 AS 级拓扑实现生成对应的 $k$ 容错拓扑需要新增加成千上万的备份链 路 (实现整个 Internet AS 级拓扑的 2 容错代价太大, 需要新增将近 3 万条备份上游链路), 我们以 1 容错拓扑为例, 通过比较 0 容错拓扑 (原始拓扑) 和 1 容错拓扑 (由原始拓扑演化而来) 在多于 $k$ 个故 障发生时的 “节点对的连通率 $\rho$ ” 对二者之间的健壮性进行比较, $\rho$ 的定义如下:

$$
\rho=\frac{M}{\frac{N(N-1)}{2}} \times 100 \%
$$

其中, $M$ 是相互可达的节点对数, $N$ 是节点总数 (不包括失效节点), $\frac{N(N-1)}{2}$ 是节点对总数.

首先, 考虑多于 $k$ 个节点故障的情况: 以增量式模式分别从 0 容错拓扑和 1 容错拓扑随机删除 $n$ 个 AS 节点, $n$ 从 100 到 $3500(\approx 10 \%)$. 如图 7(a) 所示, 1 容错拓扑对节点失效的健壮性显著优于原始 拓扑的健壮性. 同样地, 对于多于 $k$ 个链路故障的情况: 分别以增量式模式从 0 容错拓扑 (原始拓扑) 和 1 容错拓扑 (由原始拓扑演化而来) 随机删除 $n$ 条 AS 之间的连接, $n$ 从 100 到 3500. 如图 7(b) 所 


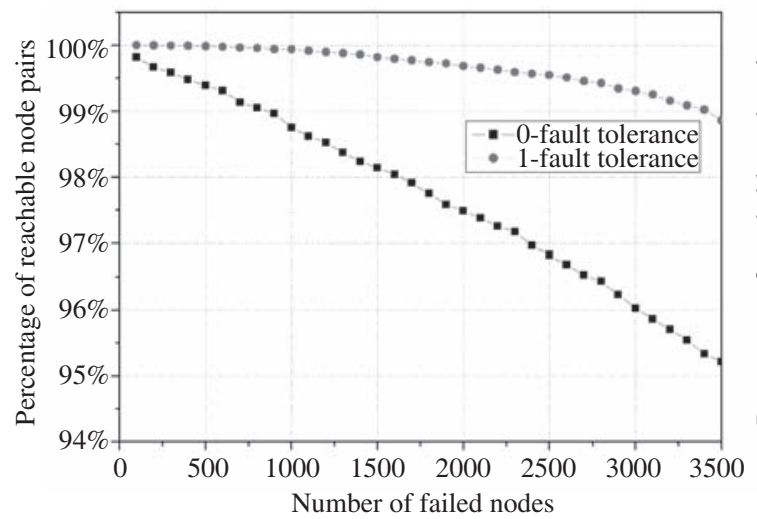

(a)

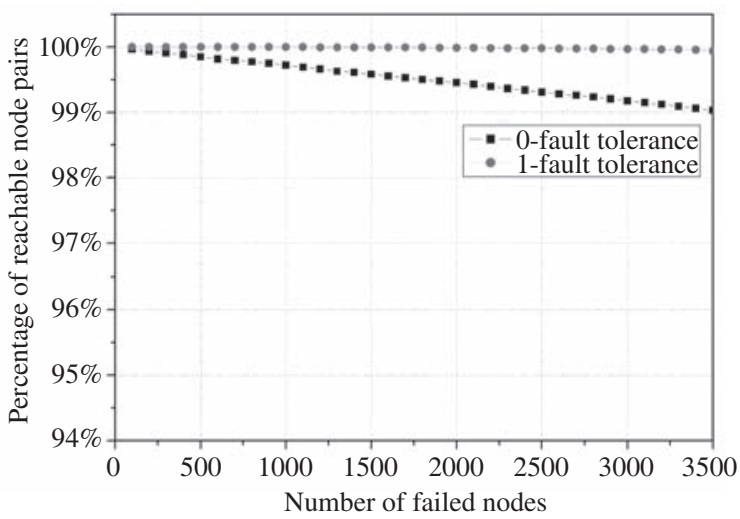

(b)

图 71 容错与 0 容错的健壮性比较

Figure 7 Robustness comparison between 1-fault tolerance and 0-fault tolerance. (a) Under node failures; (b) under link failures

示, 1 容错拓扑对链路失效的健壮性显著优于原始拓扑的健壮性. 对比图 7(a) 和 (b) 不难发现, 对于 同样的故障数目, 节点失效对拓扑健壮性的影响远远大于链路失效的影响, 因为一个 AS 节点的失效 意味着所有与该 $\mathrm{AS}$ 相连接的边也随之失效.

\section{6 结论}

本文从 AS 商业关系模型出发, 利用 Internet AS 级拓扑的层次特性, 分别从 “单个 AS”和“全局 拓扑”两个不同的层面研究了 Internet AS 级拓扑的健壮性测度与健壮性演化. 提出了对单个 AS 的 健壮性测度的方法——通过评估单个 AS 所拥有的到达顶级 AS 的节点/边不相交的上坡路径数目来 评测其对于 AS 级的节点/链路故障的健壮性. 对于全局 AS 级拓扑, 本文从域间路由系统 AS 级拓扑 的 $k$ 容错判定问题出发, 利用 AS 级拓扑固有的层次结构, 提出了 $k$ 容错模型 任意 $k$ 个 $\mathrm{AS}$ 级的 节点/链路故障不影响任何其他两个 AS 之间的连通性. 基于健壮性测度方法和 $k$ 容错模型, 提出了 健壮性演化的机制与方法. 最后, 采用 CAIDA 和 AquaLab 提供的真实拓扑数据, 对当今 Internet AS 级拓扑的健壮性进行了统计分析. 结果表明, Internet 的健壮性不容乐观, 表现在: 1) $25.8 \%$ 的 AS 节 点还不能承受 1 个 AS 级的链路故障, $26.4 \%$ 的 AS 节点还不能承受 1 个 AS 节点故障, 导致 Internet 易受区域性故障的影响, 如台风和地震的影响. 2) 当今的 Internet 距离 1 容错都还有一定的距离, 要 实现整个 AS 级拓扑的 1 容错, 需要新增 7447 条额外的上游备份链路. 实验表明, 将现有的 AS 级拓 扑演化为 $k$ 容错拓扑不仅能保证任意节点对可达性的 $k$ 容错, 在故障数超过 $k$ 的情况下, $k$ 容错拓扑 的健壮性也显著优于原拓扑.

复杂网络健壮性的一般性测度指标诸如平均路径长度、节点连通率和聚集系数等, 能从宏观上刻 画整个网络的健壮性, 但是并不能反应具体的端到端通信的健壮性, 也不能直接用于指导网络的健壮 性演化. 本文所提出的健壮性测度指标, 能够用于对整个网络健壮性的精确测度, 同时还可用于指导 整个网络拓扑的健壮性演化, 增强全网的健壮性. 


\title{
参考文献
}

1 Rekhter Y, Li T, Hares S. A border gateway protocol 4 (BGP-4). IETF RFC4271, 2006

2 Kushman N, Kandula S, Katabi D. Can you hear me now?!It must be BGP. ACM SIGCOMM CCR, 2007, 37: 77-84

3 Cowie J, Ogielski A, Premore B, et al. Impact of the 2003 blackouts on Internet communications. Technical report, Renesys Corporation, 2004

4 Kitamura Y, Lee Y, Sakiyama R, et al. Experience with restoration of Asia pacific network failures from Taiwan earthquake. IEICE T, 2007, 90: 3095-3103

5 Siganos G, Faloutsos M, Faloutsos P, et al. Powerlaws and the AS-level Internet topology. IEEE/ACM T Netw, 2003, 11: $514-524$

6 Albert R, Jeong H, Barabasi A. Attack and error tolerance of complex networks. Nature, 2000, 406: 378-382

7 Subramanian F, Agarwal S, Katz R. Characterizing the Internet hierarchy form multiple vantage points. In: Proceedings of INFOCOM. New York: IEEE, 2002. 618-627

8 Ge Z H, Figueiredo D, Jaiwal S, et al. On the hierarchical structure of the logical Internet graph. In: Proceedings of ITCOM. Denver: SPIE, 2001. 208-222

9 Gao L X. On inferring autonomous system relationships in the Internet. IEEE/ACM T Netw, 2001, 9: 733-743

10 Battista G, Erlebach T, Hall A, et al. Computing the types of the relationships between autonomous systems. IEEE/ACM T Netw, 2007, 15: 267-280

$11 \mathrm{Wu}$ J, Zhang Y, Mao Z, et al. Internet routing resilience to failures: analysis and implications. In: Proceedings of CoNEXT. New York: ACM, 2007. 1-712

12 Mühlbauer W, Feldmann A, Maennel O, et al. Building an AS-topology model that captures route diversity. In: Proceednigs of SIGCOMM. Pisa: ACM, 2006. 195-206

13 Gao L X, Rexford F, Roxford J. Stable Internet routing without global coordination. IEEE/ACM T Netw, 2001, 9: $681-692$

14 Menger K. Zur allgemeinem kurventheorie. Fund Math, 1927, 10: 96-115

15 Khuller S, Naor J. Flow in planar graphs with vertex capacities. Algorithmica, 1994, 11: 200-225

16 Lestor R, Ford F, Fulkerson D. Flows in Networks. Princeton: Princeton University Press, 1962

17 Erlebach T, Hall A, Panconesi A, et al. Cuts and disjoint paths in the valley-free path model. Int Math, 2007, 3: $333-360$

18 Erlebach T, Moonen L, Spieksma F, et al. Connectivity measures for Internet topologies on the level of autonomous systems. Operat Res, 2009, 57: 1006-1025

19 Mérindol P, Schrieck V, Donnet B, et al. Quantifying ASes multiconnectivity using multicast information. In: Proceedings of IMC. Chicago: ACM, 2009. 370-376

20 Bush R, Maennel O, Roughan M, et al. Internet optometry: assessing the broken glasses in Internet reachability. In: Proceedings of IMC. Chicago: ACM, 2009. 242-253

21 Chen K, Choffnes D, Potharaju R, et al. Where the sidewalk ends: extending the Internet AS graph using traceroutes from P2P users. In: Proceednigs of CoNEXT. Rome: ACM, 2009. 217-228

22 Deng W P, Karaliopoulos M, Mühlbauer W, et al. $k$-fault tolerance of the Internet AS graph. Comput Netw, 2011, 55: 2492-2503

\section{Robustness measurement and evolution for the Internet AS-level topology}

\author{
LU XiCheng ${ }^{1}$, DENG WenPing ${ }^{2 *}$, ZHU PeiDong ${ }^{1} \&$ HU XiaoFeng ${ }^{1}$
}


1 School of Computer, National University of Defense Technology, Changsha 410073, China;

2 Information Center, National University of Defense Technology, Changsha 410073, China

*E-mail: wpdeng.nudt@gmail.com

Abstract The inter-domain routing system consisting of autonomous systems (AS) is the key infrastructure of the Internet. It is critical to study the robustness of the Internet AS topology for the design of the robustness of the whole Internet. The Northeast Blackout (2003) and the Taiwan earthquakes (December of 2006 and March of 2010) reveal that the robustness of today's Internet still needs to be improved. Taking into account both basic theory of network topology and practical compliance to routing policies, this paper investigates the robustness measurement and evolution of the Internet AS topology at two scales: 1)we propose a method for measuring the robustness of "individual ASes" against AS link and node failures; 2)we propose a $k$-fault tolerant model for measuring the robustness of "the global AS topology"; 3)based on the two measuring methods, we present mechanisms and methods for the robustness evolution at both of the scales. Studying on today's real AS topology, we find that: 1)25.8\% of all ASes bear even single AS link failures, and $26.4 \%$ of all ASes cannot are still vulnerable to single AS node failures; 2)augmenting existing AS topology to $k$-fault tolerance cannot only guarantee the reachability of any node pairs under arbitrary $k$ failures, but also providing significantly better robustness under cases of more than $k$ failures.

Keywords inter-domain routing, autonomous system, robustness, k-fault tolerance, topology

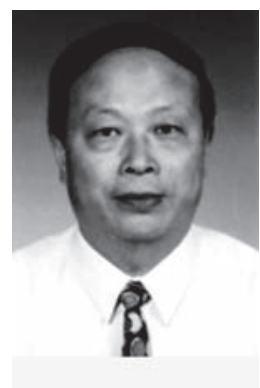

LU XiCheng received his B.S. degree in computer science from Harbin Engineering Institute, Harbin in 1970. He was a visiting scholar at the University of Massachusetts from 1982 to 1984. He is currently a Professor with School of Computer of National University of Defense Technology (NUDT), Changsha. His research interests include distributed computing, computer networks, and parallel computing. He is an academician of Chinese Academy of Engineering and a member of the IEEE.

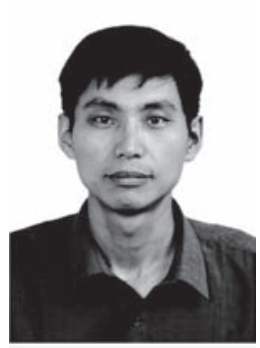

ZHU PeiDong is a Professor with School of Computer Science of $\mathrm{Na}$ tional University of Defense Technology (NUDT). He received his Ph.D. degree in computer from NUDT in 1999. From December 2008 to December 2009, he was the James visiting chair professor at St. Francis Xavier University, Canada. His research interests include network routing, network security and architecture design of the Internet and various wireless networks. He is a member of the IEEE.

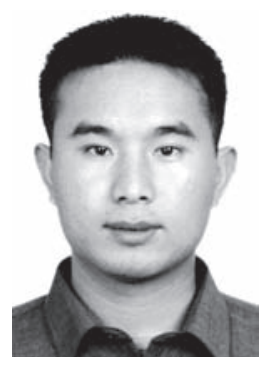

DENG WenPing received his Ph.D degree from School of Computer, National University of Defense Technology (NUDT) in 2011. He is now an Engineer at Information Center of NUDT. He was a visiting scholar to the Communication Systems Research Group (CSG) of ETH Zurich, Switzerland, from November 2008 to November 2009. His research interests include Internet routing, routing security, and resilient network.

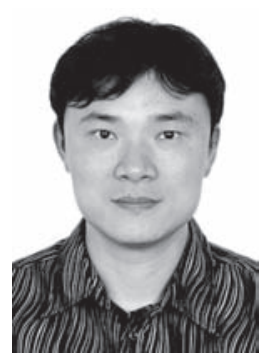

HU XiaoFeng was born in 1975. He received the Ph.D. degree in computer science and technology from $\mathrm{Na}$ tional University of Defense Technology (NUDT), Changsha in 2004. Currently, he is a Senior Researcher at School of Computer of NUDT. His research interests include Internet architecture, routing protocols, and high performance router design. 